\title{
The optical phonon spectrum of SmFeAsO
}

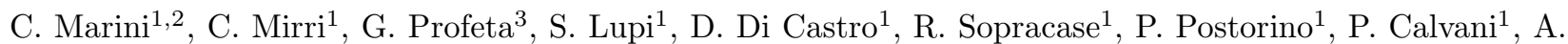 \\ Perucchi $^{4}$, S. Massidda ${ }^{5}$, G. M. Tropeano ${ }^{6}$, M. Putti ${ }^{6}$, A. Martinelli ${ }^{7}$, A. Palenzona ${ }^{7}$ and P. Dore ${ }^{1}$ \\ ${ }^{1}$ CNR-INFM "Coherentia" and Dipartimento di Fisica, \\ Universitá di Roma "La Sapienza", P.le A.Moro 2, 00185 Rome, Italy \\ 2 "Unitá CNISM Roma1" - Dipartimento di Fisica "E. Amaldi", \\ Universitá degli Studi Roma Tre, via della Vasca Navale 84,00146 Rome, Italy \\ ${ }^{3}$ CNISM - Dipartimento di Fisica, Universitá degli Studi dell'Aquila, \\ Via Vetoio 10, I-67010 Coppito (L'Aquila), Italy \\ 4 Sincrotrone Trieste S.C.p.A., S.S. $14 \mathrm{~km}$ 163.5, \\ in Area Science Park, 34012 Basovizza (Trieste), Italy \\ 5 Universitá degli Studi di Cagliari, S.P. Monserrato-Sestu km 0.700, I-09124 Monserrato (Cagliari), Italy \\ ${ }^{6}$ CNR-INFM-LAMIA and Dipartimento di Fisica, Via Dodecaneso 33, 16146 Genova, Italy and \\ 7 CNR-INFM-LAMIA and Dipartimento di Chimica e Chimica Industriale, Via Dodecaneso 31, 16146 Genova, Italy
}

(Dated: November 1, 2018)

\begin{abstract}
We measured the Raman and the Infrared phonon spectrum of SmFeAsO polycrystalline samples. We also performed Density Functional Theory calculations within the pseudopotential approximation to obtain the structural and dynamical lattice properties of both the SmFeAsO and the prototype LaFeAsO compounds. The measured Raman and Infrared phonon frequencies are well predicted by the optical phonon frequencies computed at the $\Gamma$ point, showing the capability of the employed ab-initio methods to describe the dynamical properties of these materials. A comparison among the phonon frequencies of different oxypnictides suggests a possible role of the high frequency phonons in the pairing mechanism leading to superconductivity in these materials.
\end{abstract}

PACS numbers:

\section{INTRODUCTION}

The recent discovery of high temperature superconductivity in iron-based oxypnictides [1] has triggered in the scientific community a strong research effort on this new class of superconductors. Superconducting pnictides are layered compounds containing a transition metal ( $\mathrm{Fe}, \mathrm{Co}, \mathrm{Ni}$ ) bonded with a group $\mathrm{V}$ element (As and $\mathrm{P}$ ) and intercalated with layers formed by a rare-earth $(R E=\mathrm{La}, \mathrm{Sm}, \mathrm{Nd}, \mathrm{Ce})$ and oxygen. Superconductivity appears upon doping with electrons (substitution of $\mathrm{O}$ with $\mathrm{F}$ ) or with holes (oxygen deficiency), while the parent compounds show an antiferromagnetic metallic ground state. After the discovery of superconductivity at $26 \mathrm{~K}$ in F-doped LaFeAsO [1], higher critical temperatures were soon obtained by substituting La with Sm [2], Ce [3], $\operatorname{Pr}[4]$ and Nd [5]. Also oxygen-free compounds like $A \mathrm{Fe}_{2} \mathrm{As}_{2}(A=\mathrm{Ca}, \mathrm{Sr}, \mathrm{Ba}[\underline{6}, 7])$ were found to exhibit superconductivity, even if at lower temperatures, thus opening the way to a new 'iron-age' [8].

The prototype LaFeAsO shows an anomaly near $T_{S D W}=150 \mathrm{~K}$ both in the resistivity and in the magnetic susceptibility, which is attributed to a spin-density-wave (SDW) instability [9, 10, 11]. Just above $T_{S D W}$, the system undergoes a structural transition from a high temperature tetragonal $(\mathrm{P} 4 / \mathrm{nmm})$ phase to an orthorhombic (Cmma) phase [12, 13]. It is generally recognized that doped compounds become superconducting when electron or hole doping suppresses the SDW instability [14]. However, a satisfactory theory of the superconducting mechanism in the pnictides has not yet been found. In particular, the role of phonons in the pairing mechanism has been strongly questioned due to the small value of the electron-phonon coupling 15]. The scenario is also complicated by difficulties in an ab-initio description of the normal state properties of these compounds [16]. A careful comparison between experimental and calculated properties is thus timely and important in the validation of the present first-principle theoretical methods. Structural and dynamical properties of the $R E \mathrm{FeAsO}$ compounds are an obvious ground where this comparison is due.

In this context we measured both the Raman and the Infrared (IR) phonon spectrum of pure SmFeAsO polycrystalline samples, well characterized in terms of crystal structure $(\mathrm{P} 4 / \mathrm{nmm}$ at high temperature as in $\mathrm{SmFeAsO})$, magnetic, thermal and transport properties showing that $T_{S D W} \sim 135 \div 140 \mathrm{~K}[17$, 18]. We have then calculated the phonon spectrum and compared the theoretical results with those of the experiments. 


\section{RAMAN AND INFRARED SPECTRA}

Raman spectra were measured in back-scattering geometry using a confocal micro-Raman spectrometer equipped with a He-Ne laser at $632.8 \mathrm{~nm}$. Measurements were performed on fresh-cut surfaces without any additional treatment. A camera coupled to the microscope allowed to select the point over the sample surface on which to focus the laser spot (about $5 \mu \mathrm{m}$ by using a 20X objective). Since SmFeAsO is a very poor Raman scatterer [19], spectra with a good signal-to-noise ratio were collected within the $80-1000 \mathrm{~cm}^{-1}$ range by setting long acquisition times and a large number of spectra to be averaged. It was verified that the obtained results are not affected by laser-induced sample heating. The polycrystalline nature of the sample prevent any polarization analysis of the collected spectra.

A typical room temperature Raman spectrum is shown in the inset of Fig 1, where Raman peaks are evident between 170 and $210 \mathrm{~cm}^{-1}$. Only in a few cases the collected spectra show Raman contributions due to samarium oxide 20], in agreement with the results of the analyses performed on the same sample [17]. Spectra collected from different points (P1-P4) are reported in Fig. 1 in a limited frequency range to highlight the details of the Raman peaks. Three lines are evident, at frequencies $\left(170,201\right.$ and $208 \mathrm{~cm}^{-1}$ ) which coincide with those reported in a previous room temperature Raman study which includes polarization analysis [19]. The different relative intensities of the peaks we observed in different points must be ascribed to polarization effects. Indeed the intensity of the Raman peaks can depend on the angle between the laser polarization and the possible preferred orientation of the crystallites in the scattering volume.

We also studied the temperature dependence of the Raman spectrum between 300 and $100 \mathrm{~K}$ by mounting the sample in a cryostat, which requires longer acquisition times. The comparison between the spectra collected from the same point (P4) at 300 and $100 \mathrm{~K}$ (see Fig. 1) shows no remarkable changes in the number of phonons or in the peak frequencies occurs decreasing temperature below $\mathrm{T}_{S D W}$, besides the expected phonon hardening and narrowing. The 300 and $100 \mathrm{~K}$ peak frequency values are reported in Table $\mathrm{V}$.

The infrared reflectivity $\mathrm{R}(\omega)$ of the same SmFeAsO sample was then measured from 50 to $5000 \mathrm{~cm}^{-1}$ and between 17 and $300 \mathrm{~K}$. The reference was the sample itself, gold-coated by evaporation in-situ. These measurements, performed using a Michelson interferometer at the SISSI Infrared beamline of the ELETTRA Storage Ring (Trieste, Italy), showed that $\mathrm{R}(\omega)$ is independent of temperature above $1000 \mathrm{~cm}^{-1}$. The reflectivity measurements were then extended at up to $30000 \mathrm{~cm}^{-1}$ at room temperature, by using a monochromator and an aluminum film as reference.

The inset of Fig. 2 shows that, as expected, R $(\omega)$ has a bad metallic character. A Hagen-Rubens relation was then employed for the extrapolation to $\omega=0$, usual power laws for that to $\omega=\infty$, and the optical conductivity $\sigma_{1}(\omega)$ was then obtained by standard Kramers-Kronig transformations. Due to the wide frequency range where $\mathrm{R}(\omega)$ is measured directly, $\sigma_{1}(\omega)$ in the phonon frequency range (see Fig. 2) does not depend appreciably on the choice of the extrapolations.

Although the present paper is devoted to the study of the optical phonons, it is worth noting that, in agreement with previous results on LaFeAsO [9], the optical conductivity of SmFeAsO does not provide a clear evidence of a SDW gap, indicating that not all of the Fermi surface is gapped. Only an increase of the low frequency conductivity owing to the increase of carrier mobility is observed for decreasing temperature below $T_{S D W}$ [21].

In the far-infrared spectrum five phonon peaks at 101, 259,276,377, and $448 \mathrm{~cm}^{-1}$ are evident at low temperatures. Above $150 \mathrm{~K}$, the 259 and $375 \mathrm{~cm}^{-1}$ peaks are hardly discernible and a standard Lorentzian fit was employed to determine their peak frequencies. As in the Raman case, no remarkable change in the peak frequencies occurs on decreasing temperature. The 300 and $100 \mathrm{~K}$ peak frequencies are reported in Table V. We note that the structural phase transition around $T_{S D W}$ from tetragonal to orthorhombic recently observed in $\mathrm{SmFeAsO}$ [22], as well as in LaFeAsO [12, 13] and in $\mathrm{NdFeAsO}[23]$, does not significantly affect the phonon frequencies, as also observed in the LaFeAsO case [9, 10]. However, the significant increase in the relative intensities of the 259 and $377 \mathrm{~cm}^{-1}$ peaks below $150 \mathrm{~K}$ may be regarded as an effect of the structural transition around $T_{S D W}$.

\section{CALCULATIONS}

The experimental data reported in Table V] were compared with Density Functional Theory (DFT) calculations of the SmFeAsO optical phonons, performed within the pseudopotential approximation for the electron-ion interaction 24, 25, 26]. The f-states of Sm were considered to be inside the core and a formal valence +3 was assumed for $\operatorname{Sm}\left(6 s^{2} 5 d^{1}\right.$ as valence electrons, with $5 s$ and $5 p$ treated as semi-core states $)$. The electronic density, integrated on a uniform mesh of $14 \times 14 \times 10 \mathrm{k}$-points, converged within an energy cutoff of $400 \mathrm{eV}$. A local-density approximation with gradient corrections 27] was used for the exchange correlation energy.

For comparison and cross checking with previous linear-response theory calculations, we also performed calculations for the prototype LaFeAsO compound, in the same P4/nmm structure as SmFeAsO. The theoretical lattice constants 
were obtained by minimizing the total energy as a function of the volume, after relaxing all the internal degrees of freedom. In all calculations, both systems are considered in the paramagnetic phase.

The resulting lattice constants for LaFeAsO are $\mathrm{a}=4.017 \AA, \mathrm{c}=8.677 \AA, z_{L a}=0.144$ and $z_{A s}=0.638$, in good agreement with previous computations 15 , while for $\mathrm{SmFeAsO}$ we find $\mathrm{a}=3.948 \AA, \mathrm{c}=8.336 \AA$, $z_{S m}=0.141$ and $z_{A s}=0.647$, in reasonable agreement with the experimental findings [17] (for a discussion on the agreement between experimental and computed parameters see Ref $[28,29]$ ). We notice that the smaller $\mathrm{Sm}$ ion reduces both the lattice constants: the in-plane one is reduced by about $2 \%$, while the out-of-plane one is reduced by $4 \%$. This reduction does not reflect on all interatomic bond lengths. The most affected parameter is the height of $R E$ atoms above the O plane, passing from $1.25 \AA$ in $\mathrm{LaFeAsO}$ to $1.17 \AA$ in $\mathrm{SmFeAsO}$. Consequently, the $R E$-O bond length is reduced from $2.36 \AA$ to $2.30 \AA$. The Fe-As bond lenghts instead changes from $2.34 \AA$ in LaFeAsO to $2.36 \AA$ in $\mathrm{SmFeAsO}$, although the As height above the Fe plane increases upon Sm substitution. As we will see, these structural properties are crucial to explain the differences in the phonon frequencies between these compounds.

The phonon frequencies at $\Gamma$, to be compared with the measured frequencies, were obtained by diagonalizing the dynamical matrix calculated in the frozen phonon approach 30 . For each phonon mode we report in Table $\mathrm{V}$ experimental and computed frequency, the irreducible representation associated with the mode, the polarization (X,Y: in-plane, Z: out-of-plane), the optical activity (IR or Raman), and the dominant atoms involved. A comparison with the present calculations allows one to assign all the observed phonon lines. We also report the calculated frequencies in $\mathrm{LaFeAsO}$, in good agreement with previous computations [10, 15], and the corresponding experimental values from literature data [9, 19].

A close inspection of data in Table $\nabla$ shows a good agreement (very good in some cases) between the measured and calculated frequencies. As expected, the low-frequency part of the spectrum (up to about $210 \mathrm{~cm}^{-1}$ ) is dominated by vibrations mainly involving the massive atoms $(R E$ and $A s)$. The comparison with the calculated frequencies in LaFeAsO reveals a small but systematic increase of the frequencies associated with in-plane motions. The larger $\mathrm{Sm}$ mass with respect to La would account for a slight reduction of the frequencies $\left(\omega_{S m} / \omega_{L a} \simeq\left(M_{L a} / M_{S m}\right)^{\frac{1}{2}}\right)$ in passing from La to $\mathrm{Sm}$, but this is washed out by the reduction of the strong $2 \mathrm{D}$ in-plane bonds. The same happens for the mode with Z-polarization $\left(97 \mathrm{~cm}^{-1}\right)$ that also involves the Fe-As motion. On the other hand, the modes at 177 and $203 \mathrm{~cm}^{-1}$ (out-of-plane vibrations of $R E$ and As) turn out to be softer than the respective ones in LaFeAsO, as partially confirmed by the experiments.

It is worth noting that, due to the weakness of the $R E$-As bond, the ionic interactions between the layers are weaker than the in-layer ones. We then expect that a simple rescaling of the frequencies with the $R E$ mass should be valid. This was verified by using the force constants calculated in the case of LaFeAsO (at its own lattice constants) and diagonalizing the dynamical matrix with the La atomic mass substituting that of Sm. We found that the softening observed in $\mathrm{SmFeAsO}$ is exactly predicted by this model calculation for only these two modes.

At frequencies up to $300 \mathrm{~cm}^{-1}$ the observed modes only involve the Fe-As network. The mode at $224 \mathrm{~cm}^{-1}$ involves only the Fe atoms moving along the Z-direction, and thus is expected to be unaffected by the Sm substitution and by the slight Fe-As bond reduction. The other modes, Fe-As stretching modes, are consistently higher in passing from $\mathrm{LaFeAsO}$ to $\mathrm{SmFeAsO}$.

Only in the region above $300 \mathrm{~cm}^{-1}$, which is dominated by the vibrations involving the oxygen atoms, the phonon frequencies of the two compounds are significantly different. In fact, the substitution of La with Sm produces a shortening of the $R E-\mathrm{O}$ bond length and of the $z_{R E}$ parameter (see the discussion above). This produces an overall stiffening of the $R E-\mathrm{O}$ modes; in particular, the highest frequency $\mathrm{E}_{g}$ phonon is at $434 \mathrm{~cm}^{-1}$ in LaFeAsO, at 503 $\mathrm{cm}^{-1}$ in $\mathrm{SmFeAsO}$.

\section{CONCLUSION}

We performed Raman and IR measurement on $\mathrm{SmFeAsO}$, thus obtaining accurate information on the optical phonon frequencies at the $\Gamma$ point. They show that the structural phase transition around $140 \mathrm{~K}$ does not significantly affect the phonon frequencies, as previously observed in the LaFeAsO case. We also performed a theoretical study of the structural and dynamical lattice properties by means of accurate Density Functional Theory calculations within the pseudopotential approximation. Our calculations show that, in both SmFeAsO and LaFeAsO, the Raman and IR phonon frequencies are well predicted by pseudo-potential density functional methods. This confirms the capability of ab-initio methods to describe the dynamical properties of these materials.

We find that the phonon frequencies in the two materials are very similar at low $\omega$, while those at high $\omega$ are much higher in $\mathrm{SmFeAsO}$ than in $\mathrm{LaFeAsO}$. In particular, the $\mathrm{E}_{g}$ oxygen mode is predicted to be at $434 \mathrm{~cm}^{-1}$ in LaFeAsO, at $503 \mathrm{~cm}^{-1}$ in $\mathrm{SmFeAsO}$. We have shown that this is related to the $R E$ atom being closer to the oxygen plane in $\mathrm{SmFeAsO}$ than in LaFeAsO. As a general property, the same effect can be anticipated in other oxypnictides. In fact, results on other $R E \mathrm{FeAsO}$ compounds reported in the literature show that the same mode is observed at $450 \mathrm{~cm}^{-} 1$ 
for $R E=\mathrm{Ce}[31]$, at $485 \mathrm{~cm}^{-} 1$ for $R E=\mathrm{Nd}[32]$. Bearing in mind that the superconducting transition temperature $\mathrm{T}_{c}$ of the related $\mathrm{F}$-doped systems at optimum doping depends on the $R E$ element $\left(\mathrm{T}_{c}=25 \mathrm{~K}\right.$ for $R E=\mathrm{La}[1], \mathrm{T}_{c}=40 \mathrm{~K}$ for $R E=\mathrm{Ce}[4,31], \mathrm{T}_{c}=51 \mathrm{~K}$ for $R E=\mathrm{Nd}[5]$, and $\mathrm{T}_{c}=55 \mathrm{~K}$ for $\left.R E=\mathrm{Sm}[2]\right)$ and that the phonon frequencies are not strongly affected by a low level F-doping [32], the increase of $\mathrm{T}_{c}$ appears to be correlated to the increase of the $\mathrm{E}_{g}$ phonon frequency. Although the electron-phonon coupling constant is very low for LaFeAsO [15], and probably for all oxypicnitides, the above comparison suggests that a role of the phonons in the pairing mechanism cannot be simply

ruled out, especially in view of the complex and strong magneto-phonon interaction [33] expected in these materials.

\section{ACKNOWLEDGEMENTS}

Work partially supported by the Italian Ministry of Education, through PRIN 200602174 project, by the Italian MIUR Project Cybersar (Consorzio COSMOLAB, PON 2000-2006), and by INFM-CNR through a supercomputing grant at Cineca (Bologna, Italy). One of authors (P.D.) would like to thank L. Boeri for fruitful discussions.

[1] Y. Kamihara, T. Watanabe, M. Hirano and H. Hosono, J. Am. Chem. Soc.130, 3296(2008).

[2] X. H. Chen, T. Wu, G. Wu, R. H. Liu, H. Chen and D. F. Fang, Nature 453, 761(2008).

[3] G. F. Chen, Z. Li, D. Wu, G. Li, W. Z. Hu, J. Dong, P. Zheng, J. L. Luo and N. L. Wang, Phys. Rev. Lett. 100,24700 (2008).

[4] Z. A. Ren, J. Yang, W. Lu, W. Yi, G. C. Che, X. L. Dong, L. L. Sun and Z.-X. Zhao, Materials Research Innovations 12, 105 (2008).

[5] Z. A. Ren, J. Yang, W. Lu, W. Yi, X. L. Shen, Z. C. Li, G. C. Che, X. L. Dong, L. L. Sun, F. Zhou and Z. X. Zhao, Europhys. Lett. 82,57002 (2008).

[6] M. Rotter, M. Tegel and D. Johrendt, Phys. Rev. Lett. 101, 107006 (2008).

[7] K. Sasmal, B. Lv, B. Lorenz, A. M. Guloy, F. Chen, Y. Y. Xue and C. W. Chu, Phys. Rev. Lett. 101, 107007 (2008).

[8] P. Grant, Nature 453, 1000 (2008).

[9] J. Dong, H. J. Zhang, G. Xu, Z. Li, Li G., W. Z. Hu, D. Wu, G.F. Chen, X. Dai, J. L. Luo, Z. Fang and N. L. Wang, Europhys. Lett. 83, 27006 (2008).

[10] T. Yildirim T., Phys. Rev. Lett. 101, 057010 (2008).

[11] H. H. Klauss, H. Luetkens, R. Klingeler, C. Hess, F. J. Litterst, M. Kraken, M. Korshunov, I. Eremin, S. L. Drechsler, R. Khasanov, A. Amato, J. Hamann-Borrero, N. Leps, A. Kondrat, G. Behr, J. Werner and B. Bchner, Phys. Rev. Lett. 101, 077005 (2008).

[12] C. De la Cruz, Q. Huang, J. W. Lynn, J. Li, W. Ratcliff, J. L. Zarestky, H. A. Mook, G. Chen, J. L. Luo, N. L. Wang and P. Dai, Nature 453, 899 (2008).

[13] T. Nomura, S. W. Kim, Y. Kamihara, M. Hirano, P.V. Sushko, K. Kato, M. Takata, A. L. Shluger and H. Hosono, arXiv:0804.3569 (2008).

[14] Q. Huang, J. Zhao, J. W. Lynn, G. F. Chen, J. L. Luo, N. L. Wang and P. Dai, Phys. Rev. B 78, 054529 (2008).

[15] L.Boeri, O.V. Dolgov and A. A. Golubov, Phys. Rev. Lett. 101, 026403(2008).

[16] I. I. Mazin, M. D. Johannes, L. Boeri, K. Koepernik and D. J. Singh, Phys. Rev. B 78, 085104 (2008).

[17] A. Martinelli, M. Ferretti, P. Manfrinetti, A. Palenzona, M. Tropeano, M. R. Cimberle, C. Ferdeghini, C. Valle, C. Bernini, M. Putti and A. S. Siri, Supercond. Sci. Technol. 21, 095017 (2008).

[18] M. Tropeano, A. Martinelli, A. Palenzona, E. Bellingeri, E. Galleani dAgliano, T. D. Nguyen, M. Affronte and M. Putti, Phys. Rev. B 78, 094518 (2008).

[19] V. G. Hadjiev, M. N. Iliev, K. Sasmal, Y. Y. Sun and C. W. Chu, arXiv:0804.2285(2008).

[20] T. Hongo, K. Kondo, K. Nakamura and T. Atou, J. Mater. Sci. 42, 2582 (2007).

[21] M. Tropeano, C. Fanciulli, C. Ferdeghini, D. Marr, A. S. Siri, M. Putti, A. Martinelli, M. Ferretti, A. Palenzona, M. R. Cimberle, C. Mirri, S. Lupi, R. Sopracase, P. Calvani and A. Perucchi, arXiv:0809.3500 (2008).

[22] A. Martinelli, A. Palenzona, C. Ferdeghini, M. Putti and E. Emerich arXiv:0808.1024(2008), in press on Journal of Alloys and Compounds.

[23] M. Fratini, R. Caivano, A. Puri,A. Ricci, Z. Ren, X. Dong, J. Yang, W. Lu, Z. Zhao, L. Barba, G. Arrighetti, M. Polentarutti and A. Bianconi, Supercond. Sci. Technol. 21, 092002 (2008).

[24] P. Blöchl, Phys. Rev. B 50, 17953 (1994).

[25] G. Kresse and J. Furthmüller, Phys. Rev. B 54, 11169 (1996).

[26] G. Kresse and D. Joubert, Phys. Rev. B 59, 1758 (1999).

[27] J. Perdew and Y. Wang, Phys. Rev. B 45, 13244 (1992).

[28] I.I. Mazin, M. D. Johannes, L. Boeri, K. Koepernik and D.J.Singh, arXiv0806.1869 (2008).

[29] S. Lebëgue, P. Yin and W. E. Pickett, arXiv0810.0376 (2008).

[30] D. Alfé, G. Price, and M. Gillan, Phys. Rev. B 64, 04512316 (2001). 
[31] S. C. Zhao, D. Hou, Y. Wu, T. L. Xia, A. M. Zhang, G. F. Chen, J. L. Luo, N. L. Wang, J. H. Wei, Z. Y. Lu and Q. M. Zhang, arXiv0806.0885 (2008)

[32] L.Zhang, T. Fujita, F. Chen, D. L. Feng, S. Maekawa and M. W. Chen, arXiv0809.1474 (2008)

[33] Z. Yin, S. Lebëgue, M. Han, B. Neal, S. Savrasov, and W. Pickett, Phys. Rev. Lett. 101, 047001 (2008). 


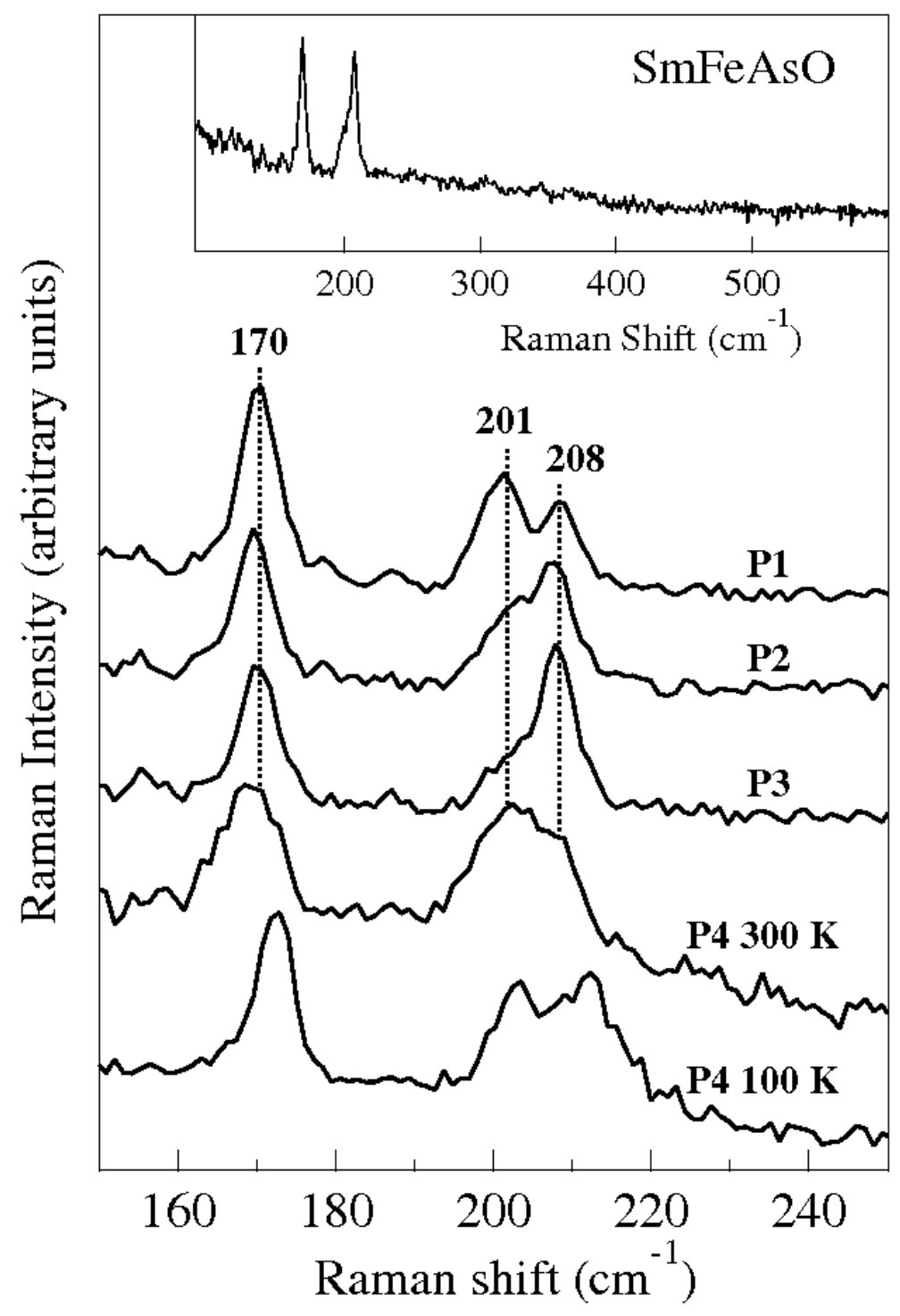

FIG. 1: Raman spectra collected between 150 and $250 \mathrm{~cm}^{-1}$ in different sample points (P1-P4) at $300 \mathrm{~K}$. The $100 \mathrm{~K}$ spectrum collected at point P4 is shown for comparison. Vertical dashed lines mark the room temperature peak frequency values. In the inset, a typical room temperature Raman spectrum of $\mathrm{SmFeAsO}$ up to $600 \mathrm{~cm}^{-1}$ is shown. 


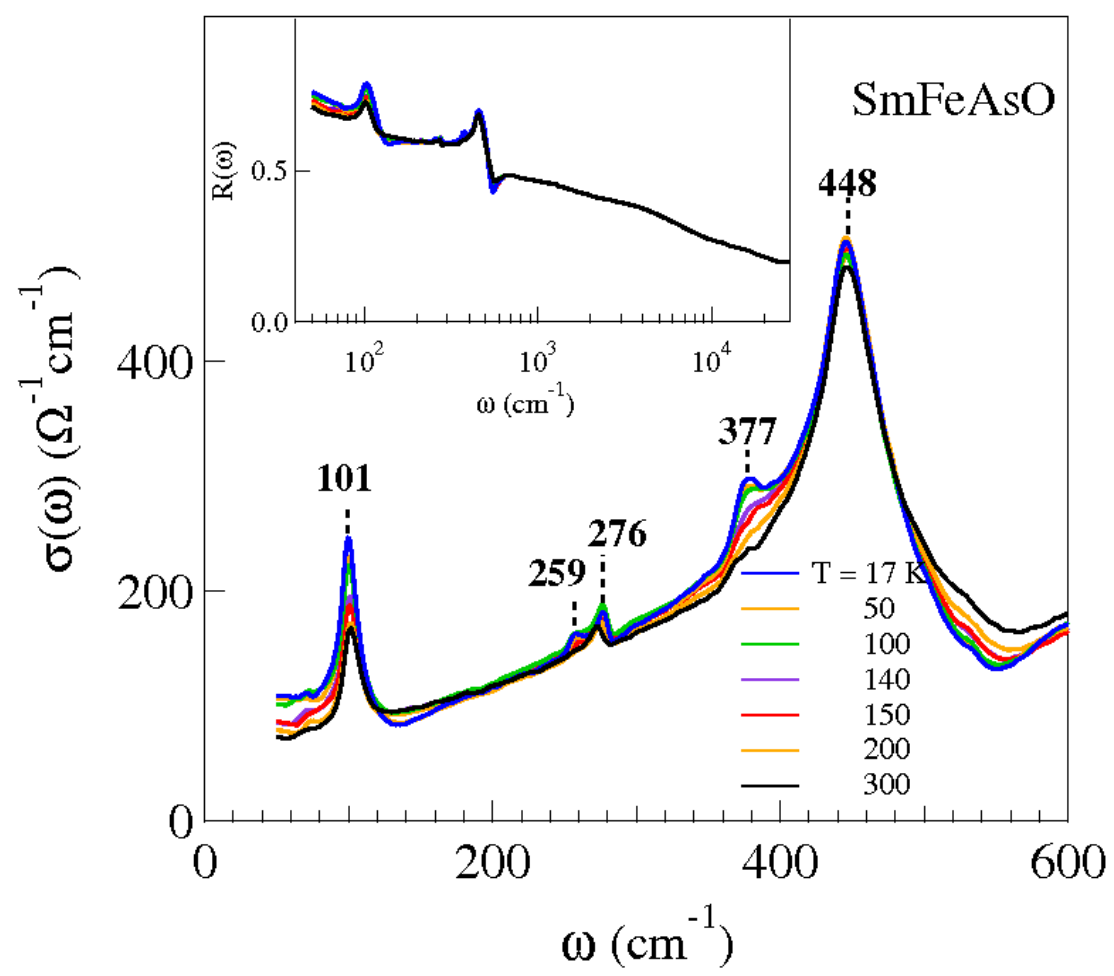

FIG. 2: (Color on line) Optical conductivity at selected temperatures in the phonon region. The $100 \mathrm{~K}$ peak frequency values are reported. The inset shows the reflectivity $\mathrm{R}(\omega)$ of $\mathrm{SmFeAsO}$ in the whole frequency range at the same temperatures 


\begin{tabular}{|c|c|c|c|c|c|c|c|}
\hline $\mathrm{SmFeAsO}$ & $\mathrm{SmFeAsO}$ & $\mathrm{LaFeAsO}$ & $\mathrm{LaFeAsO}$ & & & & Involved atoms \\
\hline Exp. & Calculated & Calculated & Exp. $[9,19]$ & Simmetry & Polarization & Activity & $(R E=\mathrm{Sm}, \mathrm{La})$ \\
\hline \multirow{4}{*}{$\begin{array}{c}101 \\
(101)\end{array}$} & 68 & 64 & & $\mathrm{Eu}$ & $\mathrm{X}, \mathrm{Y}$ & $\overline{\mathrm{IR}}$ & $R E+0+\mathrm{Fe}+\mathrm{As}$ \\
\hline & 97 & 89 & 97 & $\mathrm{~A} 2 \mathrm{u}$ & $\mathrm{Z}$ & IR & $R E+\mathrm{Fe}+\mathrm{As}$ \\
\hline & 118 & 117 & & $\mathrm{Eg}$ & $\mathrm{X}, \mathrm{Y}$ & RAM & $R E+\mathrm{As}$ \\
\hline & 148 & 144 & & $\mathrm{Eg}$ & $\mathrm{X}, \mathrm{Y}$ & RAM & $R E+\mathrm{Fe}+\mathrm{As}$ \\
\hline $\begin{array}{c}170 \\
(172)\end{array}$ & 177 & 184 & 162 & A1g & $\mathrm{Z}$ & RAM & $R E+\mathrm{As}$ \\
\hline $\begin{array}{c}201 \\
(203)\end{array}$ & 203 & 205 & 208 & A1g & $\mathrm{Z}$ & RAM & $R E+\mathrm{As}$ \\
\hline $\begin{array}{c}208 \\
(212)\end{array}$ & 224 & 224 & 201 & B1g & $\mathrm{Z}$ & RAM & $\mathrm{Fe}$ \\
\hline $\begin{array}{c}259 \\
(259)\end{array}$ & 269 & 257 & 248 & $\mathrm{~A} 2 \mathrm{u}$ & $\mathrm{Z}$ & IR & $\mathrm{Fe}+\mathrm{As}$ \\
\hline \multirow{2}{*}{$\begin{array}{c}273 \\
(276)\end{array}$} & 283 & 276 & 266 & $\mathrm{Eu}$ & $\mathrm{X}, \mathrm{Y}$ & IR & $\mathrm{Fe}+\mathrm{As}$ \\
\hline & 300 & 287 & & $\mathrm{Eg}$ & $\mathrm{X}, \mathrm{Y}$ & RAM & $\mathrm{Fe}+\mathrm{As}$ \\
\hline $345^{*}$ & 350 & 291 & 316 & B1g & $\mathrm{Z}$ & RAM & $\mathrm{O}$ \\
\hline $\begin{array}{c}377 \\
(377)\end{array}$ & 361 & 298 & 336 & $\mathrm{Eu}$ & $\mathrm{X}, \mathrm{Y}$ & IR & $R E+\mathrm{O}$ \\
\hline \multirow{2}{*}{$\begin{array}{c}446 \\
(448)\end{array}$} & 435 & 412 & 430 & $\mathrm{~A} 2 \mathrm{u}$ & $\mathrm{Z}$ & IR & $R E+\mathrm{O}+\mathrm{As}$ \\
\hline & 503 & 434 & & $\mathrm{Eg}$ & $\mathrm{X}, \mathrm{Y}$ & RAM & $\mathrm{O}$ \\
\hline
\end{tabular}

TABLE I: Room temperature peak frequencies in $\mathrm{cm}^{-1}$ for the Raman and infrared phonons of SmFeAsO, Present room temperature Raman data coincide with those of Ref. [19], where also the Raman line at $345 \mathrm{~cm}^{-1}$ (marked by *) is reported. Peak frequencies at $100 \mathrm{~K}$ are given in brackets. Calculated phonon frequencies are given for SmFeAsO and LaFeAsO, the latter compared with literature experimental values from Refs. [9, 19]. Mode symmetry, polarization (X,X: in-plane, Z: out-of-plane), optical activity (IR or Raman) and dominant atoms involved in each mode are also given. 